\title{
Supersymmetric Yang-Mills theory in $D=6$ without anticommuting variables
}

\author{
Sudarshan Ananth $\odot,{ }^{1}$ Hannes Malcha $\odot,{ }^{2}$ Chetan Pandey, ${ }^{1}$ and Saurabh Pant $\odot^{1}$ \\ ${ }^{1}$ Indian Institute of Science Education and Research Pune 411008, India \\ ${ }^{2}$ Max-Planck-Institut für Gravitationsphysik (Albert-Einstein-Institut) Am Mühlenberg 1, \\ 14476 Potsdam, Germany
}

(Received 21 September 2020; accepted 4 January 2021; published 12 January 2021)

\begin{abstract}
Supersymmetric Yang-Mills theory is formulated in six dimensions, without the use of anticommuting variables. This is achieved using a new Nicolai map, to third order in the coupling constant. This is the second such map in six dimensions and highlights a potential ambiguity in the formalism.
\end{abstract}

DOI: 10.1103/PhysRevD.103.025010

\section{INTRODUCTION AND NOTATION}

Supersymmetric theories may be formulated without the use of anticommuting variables [1,2]. In this approach, supersymmetric gauge theories are characterized by a Nicolai map - a transformation of the bosonic fields such that the Jacobian determinant of the transformation exactly cancels against the product of the Matthews-Salam-Seiler (MSS) $[3,4]$ and Faddeev-Popov (FP) determinants [5,6]. This formalism offers an alternate perspective on the physics of supersymmetric gauge theories.

The Nicolai map, for Yang-Mills theory, was explicitly constructed to second order in the coupling constant in [7] and derived from a rigorous $\mathcal{R}$ prescription in [8-11]. It was subsequently shown [12] that this construction holds in all the critical dimensions $D=3,4,6,10$ where supersymmetric YangMills theories exist [13]. The map and the framework itself were extended to third order in the coupling constant in [14].

In this paper, we present a stand-alone result-a new map, also to third order in the coupling constant, but valid exclusively in six dimensions. The map presented here, arrived at by trial and error (starting with an educated guess), is simpler than the one in [14]. This highlights a potential ambiguity in the $\mathcal{R}$ prescription approach, an aspect of which was also discussed in [14]. For $\mathcal{N}=1$ Yang Mills theories, alternate Nicolai maps are known to exist in four and six dimensions $[15,16]$. These maps in light cone gauge, not constructed from a $\mathcal{R}$ prescription, work to all orders in perturbation theory.

Published by the American Physical Society under the terms of the Creative Commons Attribution 4.0 International license. Further distribution of this work must maintain attribution to the author(s) and the published article's title, journal citation, and DOI. Funded by SCOAP.
Supersymmetric gauge theories, in $D$ dimensions, are characterized by the existence of a Nicolai map $\mathcal{T}_{g}$ of the Yang-Mills fields

$$
\mathcal{T}_{g}: A_{\mu}^{a}(x) \mapsto A_{\mu}^{\prime a}(x, g ; A),
$$

such that

(i) The Yang-Mills action without gauge-fixing terms is mapped to the Abelian action

$$
S_{0}\left[A^{\prime}\right]=S_{g}[A]
$$

where $S_{g}[A]=\frac{1}{4} \int \mathrm{d} x F_{\mu \nu}^{a} F_{\mu \nu}^{a}$ is the Yang-Mills action with gauge coupling $g$ and $F_{\mu \nu}^{a} \equiv \partial_{\mu} A_{\nu}^{a}-$ $\partial_{\nu} A_{\mu}^{a}+g f^{a b c} A_{\mu}^{b} A_{\nu}^{c}$ is the field strength.

(ii) On the gauge surface ${ }^{1} G^{a}[A] \equiv \partial^{\mu} A_{\mu}^{a}=0$, the Jacobi determinant of $\mathcal{T}_{g}$ is equal to the product of the MSS and FP determinants, order by order in perturbation theory.

$$
\operatorname{det}\left(\frac{\delta A_{\mu}^{\prime a}(x, g ; A)}{\delta A_{\nu}^{b}(y)}\right)=\Delta_{M S S}[A] \Delta_{F P}[A] .
$$

(iii) The gauge fixing function

$$
G^{a}[A] \text { is a fixed point of } \mathcal{T}_{g} .
$$

A new expression for $A_{\mu}^{\prime a}(x)$ up to order $g^{3}$ is presented in this paper and shown to satisfy all three requirements above only in $D=6$.

\footnotetext{
${ }^{1}$ The gauge surface restriction will prove unnecessary for this particular map.
} 
We work in Euclidean space using the Landau gauge

$$
G^{a}\left[A_{\mu}\right]=\partial^{\mu} A_{\mu}^{a}
$$

The results presented below may be adapted to other gauges (the light-cone gauge being of particular interest given potential links to $[17,18])$. The free scalar propagator is $\left(\square \equiv \partial^{\mu} \partial_{\mu}\right)$

$$
C(x)=\int \frac{d^{D} k}{(2 \pi)^{D}} \frac{e^{i k x}}{k^{2}} \Rightarrow-\square C(x)=\delta(x)
$$

The free fermion propagator is (spinor indices suppressed)

$$
\gamma^{\mu} \partial_{\mu} S_{0}(x)=\delta(x) \Rightarrow S_{0}(x)=-\gamma^{\mu} \partial_{\mu} C(x)
$$

$S_{0}(x-y)=-S_{0}(y-x)$. In a gauge-field dependent background

$\gamma^{\mu}\left(D_{\mu} S\right)^{a b}(x) \equiv \gamma^{\mu}\left[\delta^{a c} \partial_{\mu}-g f^{a c d} A_{\mu}^{d}(x)\right] S^{c b}(x)=\delta^{a b} \delta(x)$.

\section{RESULT}

The new result in this paper is the following explicit expression for $\mathcal{T}_{g}$ to $\mathcal{O}\left(g^{3}\right)$.

$$
\begin{aligned}
\left(\mathcal{T}_{g} A\right)_{\mu}^{a}(x)= & A_{\mu}^{a}(x)+g f^{a b c} \int d y \partial_{\lambda} C(x-y) A_{\mu}^{b}(y) A_{\lambda}^{c}(y)+\frac{3}{2} g^{2} f^{a b c} f^{b d e} \int d y d z \partial_{\rho} C(x-y) A_{\sigma}^{c}(y) \partial_{[\rho} C(y-z) A_{\mu}^{d}(z) A_{\sigma]}^{e}(z) \\
& +\frac{3}{2} g^{3} f^{a b c} f^{b d e} f^{d m n} \int d y d z d w \partial_{\rho} C(x-y) A_{\lambda}^{c}(y)\left\{+\partial_{\lambda} C(y-z) A_{\sigma}^{e}(z) \partial_{[\mu} C(z-w) A_{\rho}^{m}(w) A_{\sigma]}^{n}(w)\right. \\
& \left.+\partial_{\mu} C(y-z) A_{\sigma}^{e}(z) \partial_{[\sigma} C(z-w) A_{\rho}^{m}(w) A_{\lambda]}^{n}(w)+\partial_{\rho} C(y-z) A_{\sigma}^{e}(z) \partial_{[\sigma} C(z-w) A_{\lambda}^{m}(w) A_{\mu]}^{n}(w)\right\} \\
& -g^{3} f^{a b c} f^{b d e} f^{d m n} \int d y d z d w \partial_{\rho} C(x-y) A_{\lambda}^{c}(y)\left\{+\partial_{\sigma} C(y-z) A_{\sigma}^{e}(z) \partial_{[\mu} C(z-w) A_{\lambda}^{m}(w) A_{\rho]}^{n}(w)\right. \\
& +\partial_{\sigma} C(y-z) A_{\rho}^{e}(z) \partial_{[\sigma} C(z-w) A_{\lambda}^{m}(w) A_{\mu]}^{n}(w)+\partial_{\sigma} C(y-z) A_{\mu}^{e}(z) \partial_{[\sigma} C(z-w) A_{\rho}^{m}(w) A_{\lambda]}^{n}(w) \\
& \left.+\partial_{\sigma} C(y-z) A_{\lambda}^{e}(z) \partial_{[\mu} C(z-w) A_{\rho}^{m}(w) A_{\sigma]}^{n}(w)\right\},
\end{aligned}
$$

where $[\mu \nu \rho]=\frac{1}{6}[\mu \nu \rho-\mu \rho \nu+\nu \rho \mu-\nu \mu \rho+\rho \mu \nu-\rho \nu \mu]$.

It is important to note that this result differs from the one in [14]. All terms above have the base structure $\partial C A \partial C A \partial C A A$ at $\mathcal{O}\left(g^{3}\right)$, while the result in [14] also includes the structures $\partial C \partial C A A \partial C A A, A C A \partial C A A$, and $\partial C \partial(A C) A \partial C A A$.

Further, terms that overlap with those in [14], appear here with different coefficients. As a consequence, the expression above is not a subset of the result in [14].

Finally, while the result in [14] was valid in all the critical dimensions, we will see that the result in (8) constitutes a map only in six dimensions.

\section{CHECKS OF THE RESULT}

In this section, we prove that expression in (8) satisfies all three requirements, (1), (2), and (3), necessary for it to be a map. The calculations up to $\mathcal{O}\left(g^{2}\right)$ are identical to those in $[12,14]$, so the focus here will be on $\mathcal{O}\left(g^{3}\right)$.

\section{A. Gauge condition}

We begin with the third requirement, listed in (3). We need to show that $\partial_{\mu} A_{\mu}^{\prime a}(x)=\partial_{\mu} A_{\mu}^{a}(x)+\mathcal{O}\left(g^{4}\right)$.

We apply $\partial_{\mu}$ to the terms of order $g^{3}$ in (8). This gives us a symmetric $\partial_{\mu} \partial_{\rho}$ at the beginning of the expression so we eliminate all terms that are antisymmetric under the exchange $\mu \leftrightarrow \rho$ and find

$$
\begin{aligned}
\left.\partial_{\mu} A_{\mu}^{\prime a}(x)\right|_{\mathcal{O}\left(g^{3}\right)}= & \frac{3}{2} g^{3} f^{a b c} f^{b d e} f^{d m n} \int d y d z d w \partial_{\mu} \partial_{\rho} C(x-y) A_{\lambda}^{c}(y)\left\{+\partial_{\mu} C(y-z) A_{\sigma}^{e}(z) \partial_{[\sigma} C(z-w) A_{\rho}^{m}(w) A_{\lambda]}^{n}(w)\right. \\
& \left.+\partial_{\rho} C(y-z) A_{\sigma}^{e}(z) \partial_{[\sigma} C(z-w) A_{\lambda}^{m}(w) A_{\mu]}^{n}(w)\right\}-g^{3} f^{a b c} f^{b d e} f^{d m n} \int d y d z d w \partial_{\mu} \partial_{\rho} C(x-y) A_{\lambda}^{c}(y) \\
& \times\left\{+\partial_{\sigma} C(y-z) A_{\rho}^{e}(z) \partial_{[\sigma} C(z-w) A_{\lambda}^{m}(w) A_{\mu]}^{n}(w)+\partial_{\sigma} C(y-z) A_{\mu}^{e}(z) \partial_{[\sigma} C(z-w) A_{\rho}^{m}(w) A_{\lambda]}^{n}(w)\right\} .
\end{aligned}
$$

The first two terms cancel each other under the interchange of $\mu$ and $\rho$. Similarly, the other two terms also cancel out confirming that

$$
\partial_{\mu} A_{\mu}^{\prime a}(x)=\partial_{\mu} A_{\mu}^{a}(x)+\mathcal{O}\left(g^{4}\right)
$$




\section{B. Free action}

We now move to the first requirement in (1) which states that the transformed gauge field must satisfy

$$
\frac{1}{2} \int d x A_{\mu}^{\prime a}(x)\left(-\square \delta_{\mu \nu}+\partial_{\mu} \partial_{\nu}\right) A_{\nu}^{\prime a}(x)=\frac{1}{4} \int d x F_{\mu \nu}^{a}(x) F_{\mu \nu}^{a}(x)+\mathcal{O}\left(g^{4}\right) .
$$

Because of the invariance of the gauge function, we ignore the second term on the left-hand side (lhs) and the corresponding term on the right-hand side (rhs) of this equation [12]. At third order, (11) has two contributions

$$
0 \stackrel{!}{=} \int d x\left(\left.\left.A_{\mu}^{\prime a}(x)\right|_{\mathcal{O}\left(g^{3}\right)} \square A_{\mu}^{\prime a}(x)\right|_{\mathcal{O}\left(g^{0}\right)}+\left.\left.A_{\mu}^{\prime a}(x)\right|_{\mathcal{O}\left(g^{2}\right)} \square A_{\mu}^{\prime a}(x)\right|_{\mathcal{O}\left(g^{1}\right)}\right) .
$$

This expression reads

$$
\begin{array}{rl}
\int d & x\left(\left.\left.A_{\mu}^{\prime a}(x)\right|_{\mathcal{O}\left(g^{3}\right)} \square A_{\mu}^{\prime a}(x)\right|_{\mathcal{O}\left(g^{0}\right)}+\left.\left.A_{\mu}^{\prime a}(x)\right|_{\mathcal{O}\left(g^{2}\right)} \square A_{\mu}^{\prime a}(x)\right|_{\mathcal{O}\left(g^{1}\right)}\right) \\
= & \frac{3}{2} g^{3} f^{a b c} f^{b d e} f^{d m n} \int d x d y d z d w \partial_{\rho} C(x-y) A_{\lambda}^{c}(y)\left\{+\partial_{\lambda} C(y-z) A_{\sigma}^{e}(z) \partial_{[\mu} C(z-w) A_{\rho}^{m}(w) A_{\sigma]}^{n}(w) \square A_{\mu}^{a}(x)\right. \\
& \left.+\partial_{\mu} C(y-z) A_{\sigma}^{e}(z) \partial_{[\sigma} C(z-w) A_{\rho}^{m}(w) A_{\lambda]}^{n}(w) \square A_{\mu}^{a}(x)+\partial_{\rho} C(y-z) A_{\sigma}^{e}(z) \partial_{[\sigma} C(z-w) A_{\lambda}^{m}(w) A_{\mu]}^{n}(w) \square A_{\mu}^{a}(x)\right\} \\
& -g^{3} f^{a b c} f^{b d e} f^{d m n} \int d x d y d z d w \partial_{\rho} C(x-y) A_{\lambda}^{c}(y)\left\{+\partial_{\sigma} C(y-z) A_{\sigma}^{e}(z) \partial_{[\mu} C(z-w) A_{\lambda}^{m}(w) A_{\rho]}^{n}(w) \square A_{\mu}^{a}(x)\right. \\
& +\partial_{\sigma} C(y-z) A_{\rho}^{e}(z) \partial_{[\sigma} C(z-w) A_{\lambda}^{m}(w) A_{\mu]}^{n}(w) \square A_{\mu}^{a}(x)+\partial_{\sigma} C(y-z) A_{\mu}^{e}(z) \partial_{[\sigma} C(z-w) A_{\rho}^{m}(w) A_{\lambda]}^{n}(w) \square A_{\mu}^{a}(x) \\
& \left.+\partial_{\sigma} C(y-z) A_{\lambda}^{e}(z) \partial_{[\mu} C(z-w) A_{\rho}^{m}(w) A_{\sigma]}^{n}(w) \square A_{\mu}^{a}(x)\right\} \\
& +\frac{3}{2} g^{3} f^{a b c} f^{b d e} \int d x d y d z d w \partial_{\rho} C(x-y) A_{\lambda}^{c}(y) \partial_{[\rho} C(y-z) A_{\mu}^{d}(z) A_{\lambda]}^{e}(z) \square\left(f^{a m n} \partial_{\sigma} C(x-w) A_{\mu}^{m}(w) A_{\sigma}^{n}(w)\right) .
\end{array}
$$

We simplify the rhs to obtain

$$
\begin{aligned}
= & \frac{3}{2} g^{3} f^{a b c} f^{b d e} f^{d m n} \int d x d z d w \partial_{\rho} A_{\mu}^{a}(x) A_{\lambda}^{c}(x)\left\{+\partial_{\lambda} C(x-z) A_{\sigma}^{e}(z) \partial_{[\mu} C(z-w) A_{\rho}^{m}(w) A_{\sigma]}^{n}(w)\right. \\
& \left.+\partial_{\mu} C(x-z) A_{\sigma}^{e}(z) \partial_{[\sigma} C(z-w) A_{\rho}^{m}(w) A_{\lambda]}^{n}(w)+\partial_{\rho} C(x-z) A_{\sigma}^{e}(z) \partial_{[\sigma} C(z-w) A_{\lambda}^{m}(w) A_{\mu]}^{n}(w)\right\} \\
& -g^{3} f^{a b c} f^{b d e} f^{d m n} \int d x d z d w \partial_{\rho} A_{\mu}^{a}(x) A_{\lambda}^{c}(x)\left\{+\partial_{\sigma} C(x-z) A_{\sigma}^{e}(z) \partial_{[\mu} C(z-w) A_{\lambda}^{m}(w) A_{\rho]}^{n}(w)\right. \\
& +\partial_{\sigma} C(x-z) A_{\rho}^{e}(z) \partial_{[\sigma} C(z-w) A_{\lambda}^{m}(w) A_{\mu]}^{n}(w)+\partial_{\sigma} C(x-z) A_{\mu}^{e}(z) \partial_{[\sigma} C(z-w) A_{\rho}^{m}(w) A_{\lambda]}^{n}(w) \\
& \left.+\partial_{\sigma} C(x-z) A_{\lambda}^{e}(z) \partial_{[\mu} C(z-w) A_{\rho}^{m}(w) A_{\sigma]}^{n}(w)\right\} \\
& +\frac{3}{2} g^{3} f^{a b c} f^{b d e} f^{a m n} \int d x d z d w A_{\lambda}^{c}(x) \partial_{[\rho} C(x-z) A_{\mu}^{d}(z) A_{\lambda]}^{e}(z) \partial_{\rho} \partial_{\sigma} C(x-w) A_{\mu}^{m}(w) A_{\sigma}^{n}(w) .
\end{aligned}
$$

This is further simplified with some rewriting [for example, $\partial_{\rho} A_{\mu}^{a}(x) A_{\lambda}^{c}(x) \rightarrow \frac{1}{2} \partial_{\rho}\left(A_{\mu}^{a}(x) A_{\lambda}^{c}(x)\right)$ based on the symmetries $a \leftrightarrow c$ and $\mu \leftrightarrow \lambda]$. The rhs simplifies to

$$
=\frac{3}{4} g^{3} f^{a b c} f^{b d e} f^{d m n} A_{\mu}^{a}(x) A_{\lambda}^{c}(x) A_{\sigma}^{e}(x) \partial_{[\sigma} C(x-w) A_{\lambda}^{m}(w) A_{\mu]}^{n}(w) .
$$

There is a symmetry to these terms: the $\partial C A A$ blocks are invariant under a cyclic permutation of the Lorentz indices. This motivates rewriting the term as

$$
\begin{gathered}
\frac{1}{4} g^{3} f^{a b c} f^{b d e} f^{d m n}\left[A_{\mu}^{a}(x) A_{\lambda}^{c}(x) A_{\sigma}^{e}(x)+A_{\sigma}^{a}(x) A_{\mu}^{c}(x) A_{\lambda}^{e}(x)+A_{\lambda}^{a}(x) A_{\sigma}^{c}(x) A_{\mu}^{e}(x)\right] \partial_{[\sigma} C(x-w) A_{\lambda}^{m}(w) A_{\mu]}^{n}(w) \\
=\frac{1}{4} g^{3}\left[f^{a b c} f^{b d e}+f^{e b a} f^{b d c}+f^{c b e} f^{b d a}\right] f^{d m n} A_{\mu}^{a}(x) A_{\lambda}^{c}(x) A_{\sigma}^{e}(x) \partial_{[\sigma} C(x-w) A_{\lambda}^{m}(w) A_{\mu]}^{n}(w) .
\end{gathered}
$$


We now find, for the first time in this computation, that for (14) to vanish we need to invoke the Jacobi identity

$$
f^{a b c} f^{b d e}+f^{e b a} f^{b d c}+f^{c b e} f^{b d a}=0 .
$$

Thus (11) holds up to $\mathcal{O}\left(g^{3}\right)$.

\section{Jacobians, fermion, and ghost determinants}

Finally, we turn to (2), the second requirement. This is, in some sense, the most constraining of the three requirements, demanding that the bosonic Jacobian determinant equal the product of the MSS and FP determinants. Again, this check up to $\mathcal{O}\left(g^{2}\right)$ was performed in $[7,12]$ allowing us to concentrate here on $\mathcal{O}\left(g^{3}\right)$.

$\left.\left.\log \operatorname{det}\left(\frac{\delta A_{\mu}^{\prime a}(x)}{\delta A_{\nu}^{b}(y)}\right)\right|_{\mathcal{O}\left(g^{3}\right)} \stackrel{!}{=} \log \left(\Delta_{M S S}[A] \Delta_{F P}[A]\right)\right|_{\mathcal{O}\left(g^{3}\right)}$.

It is this nontrivial requirement which results in a dimensional dependence. We prove that the map in (8) satisfies (16) only for $D=6$.

\section{Fermion determinant}

To compute the fermion determinant, we need to evaluate the following quantity

$$
\operatorname{det}\left[\gamma^{\mu}\left(\delta^{a b} \partial_{\mu}-g f^{a b m} A_{\mu}^{m}\right)\right]=\operatorname{det} \not \partial \cdot \operatorname{det}(1-Y),
$$

where the relevant functional matrix reads

$$
Y^{a b}(x, y ; A)=g f^{a b m} \gamma^{\mu} \gamma^{\nu} \partial_{\mu} C(x-y) A_{\nu}^{m}(y) .
$$

For the treatment of the $\gamma$-matrices we only need the Clifford algebra relation $\left\{\gamma_{\mu}, \gamma_{\nu}\right\}=2 \delta_{\mu \nu}$ and the trace

$$
\operatorname{Tr} \mathbf{1}=r,
$$

where $r$ counts the number of off-shell fermionic degrees of freedom. These relations fully define the trace of the product of any number of $\gamma$-matrices and it will not be necessary to explicitly distinguish between Majorana, Weyl, and Majorana-Weyl spinors. Even though $r$ is related to the number of dimensions $D$ via

$$
r=2(D-2)
$$

we will treat them as independent for now. The relation above is only invoked at the very last step, to highlight the crucial fact that the Nicolai map presented here only works in six dimensions. Furthermore, we use

$$
\log \operatorname{det}(1-Y)=\operatorname{Tr} \log (1-Y)=-\sum_{n=1}^{\infty} \frac{1}{n} \operatorname{Tr} Y^{n},
$$

and taking into account an extra factor of $\frac{1}{2}$ for the on-shell degrees of freedom, we find

$\frac{1}{2} \log \operatorname{det}(\mathbf{1}-\mathbf{Y})=\frac{1}{6} g^{3} \operatorname{Tr}\left(\gamma^{\alpha} \gamma^{\beta} \gamma^{\rho} \gamma^{\sigma} \gamma^{\lambda} \gamma^{\tau}\right) f^{a b m} f^{b c n} f^{c a p} \int d x d y d z\left\{\partial_{\alpha} C(x-y) A_{\beta}^{m}(y) \partial_{\rho} C(y-z) A_{\sigma}^{n}(z) \partial_{\lambda} C(z-x) A_{\tau}^{p}(x)\right\}$,

From the relations above the trace over six $\gamma$-matrices can be computed recursively and reads

$$
\begin{aligned}
\operatorname{Tr}\left(\gamma^{\alpha} \gamma^{\beta} \gamma^{\rho} \gamma^{\sigma} \gamma^{\lambda} \gamma^{\tau}\right)= & -r\left(\delta^{\alpha \beta} \delta^{\lambda \tau} \delta^{\rho \sigma}-\delta^{\alpha \beta} \delta^{\lambda \sigma} \delta^{\rho \tau}+\delta^{\alpha \beta} \delta^{\lambda \rho} \delta^{\sigma \tau}+\delta^{\alpha \lambda} \delta^{\beta \tau} \delta^{\rho \sigma}-\delta^{\alpha \lambda} \delta^{\beta \sigma} \delta^{\rho \tau}+\delta^{\alpha \lambda} \delta^{\beta \rho} \delta^{\sigma \tau}+\delta^{\alpha \sigma} \delta^{\beta \lambda} \delta^{\rho \tau}+\delta^{\alpha \rho} \delta^{\beta \tau} \delta^{\lambda \sigma}\right. \\
& \left.+\delta^{\alpha \rho} \delta^{\beta \sigma} \delta^{\lambda \tau}-\delta^{\alpha \rho} \delta^{\beta \lambda} \delta^{\sigma \tau}+\delta^{\alpha \tau} \delta^{\beta \sigma} \delta^{\lambda \rho}-\delta^{\alpha \sigma} \delta^{\beta \tau} \delta^{\lambda \rho}-\delta^{\alpha \sigma} \delta^{\beta \rho} \delta^{\lambda \tau}-\delta^{\alpha \tau} \delta^{\beta \rho} \delta^{\lambda \sigma}-\delta^{\alpha \tau} \delta^{\beta \lambda} \delta^{\rho \sigma}\right)
\end{aligned}
$$

Equation (22) then yields the following five independent terms at order $g^{3}$

$$
\begin{aligned}
& g^{3} f^{a b m} f^{b c n} f^{c a p} \int d x d y d z\left\{-r \partial_{\rho} C(x-y) A_{\rho}^{m}(y) \partial_{\lambda} C(y-z) A_{\sigma}^{n}(z) \partial_{\lambda} C(z-x) A_{\sigma}^{p}(x)\right. \\
& \quad+\frac{r}{3} \partial_{\rho} C(x-y) A_{\lambda}^{m}(y) \partial_{\lambda} C(y-z) A_{\sigma}^{n}(z) \partial_{\sigma} C(z-x) A_{\rho}^{p}(x) \\
& \quad+\frac{r}{2} \partial_{\rho} C(x-y) A_{\lambda}^{m}(y) \partial_{\lambda} C(y-z) A_{\rho}^{n}(z) \partial_{\sigma} C(z-x) A_{\sigma}^{p}(x) \\
& \quad-\frac{r}{6} \partial_{\rho} C(x-y) A_{\lambda}^{m}(y) \partial_{\sigma} C(y-z) A_{\rho}^{n}(z) \partial_{\lambda} C(z-x) A_{\sigma}^{p}(x) \\
& \left.\quad+\frac{r}{2} \partial_{\rho} C(x-y) A_{\lambda}^{m}(y) \partial_{\sigma} C(y-z) A_{\rho}^{n}(z) \partial_{\sigma} C(z-x) A_{\lambda}^{p}(x)\right\}
\end{aligned}
$$




\section{Ghost determinant}

For the ghost determinant, we compute

$$
\operatorname{det}\left(D_{\mu} \partial^{\mu}\right)=\operatorname{det}\left(\left[\delta^{a b} \partial_{\mu}-g f^{a b m} A_{\mu}^{m}\right] \partial^{\mu}\right)=\operatorname{det}(\square) \cdot \operatorname{det}(1-X),
$$

where

$$
X^{a b}(x, y ; A)=g f^{a b m} \partial_{\mu} C(x-y) A_{\mu}^{m}(y) .
$$

Up to $\mathcal{O}\left(g^{3}\right)$ this yields

$$
+\frac{1}{3} g^{3} f^{a b m} f^{b c n} f^{c a p} \int d x d y d z \partial_{\rho} C(x-y) A_{\rho}^{m}(y) \partial_{\lambda} C(y-z) A_{\lambda}^{n}(z) \partial_{\sigma} C(z-x) A_{\sigma}^{p}(x) .
$$

\section{Bosonic Jacobian}

At $\mathcal{O}\left(g^{3}\right)$ the logarithm of the Jacobian determinant schematically consists of three terms

$$
\left.\log \operatorname{det}\left(\frac{\delta A_{\mu}^{\prime a}(x)}{\delta A_{\nu}^{b}(y)}\right)\right|_{\mathcal{O}\left(g^{3}\right)}=\operatorname{Tr}\left[\left.\frac{\delta A^{\prime}}{\delta A}\right|_{\mathcal{O}\left(g^{3}\right)}\right]-\left(2 \cdot \frac{1}{2}\right) \operatorname{Tr}\left[\left.\left.\frac{\delta A^{\prime}}{\delta A}\right|_{\mathcal{O}\left(g^{2}\right)} \frac{\delta A^{\prime}}{\delta A}\right|_{\mathcal{O}\left(g^{1}\right)}\right]+\frac{1}{3} \operatorname{Tr}\left[\left.\left.\left.\frac{\delta A^{\prime}}{\delta A}\right|_{\mathcal{O}\left(g^{1}\right)} \frac{\delta A^{\prime}}{\delta A}\right|_{\mathcal{O}\left(g^{1}\right)} \frac{\delta A^{\prime}}{\delta A}\right|_{\mathcal{O}\left(g^{1}\right)}\right]
$$

and the final trace involves setting $\mu=\nu, a=b, x=y$ and integrating over $x$.

All terms at $\mathcal{O}\left(g^{3}\right)$ are of the form $\partial C A \partial C A \partial C A A$. The functional derivative on the very first field, in this structure, vanishes trivially [12]. The functional differentiation of the field in the middle block produces the structure $\partial C A \partial C \partial C A A$ not seen elsewhere. These terms vanish as described in the Appendix. Functional differentiation of either field from the last block produces terms with the same structure as those from the fermion and ghost contributions. The table below offers a summary of the various contributions to the Jacobian from (28).

\section{Jacobian table}

In the table, columns 2-5 capture bosonic contributions, summed up in column 6 . Column 7 contains the sums of the fermion and ghost contributions. The detailed breakdown for the bosonic contributions is as follows: Column 2 contains the contributions from $\mathcal{O}(g)$ terms when "cubed." Column 3 lists contributions from $\mathcal{O}(g) \times \mathcal{O}\left(g^{2}\right)$. Column 4 has contributions from the 9 terms in the bosonic result [first three lines of $\mathcal{O}\left(g^{3}\right)$ from (8)]. In column 5, we present contributions from the next four lines of (8) (12 terms).

In column 7, we now set [12]

$$
r=2(D-2)
$$

The main result is that columns 6 and 7 are equal only

\begin{tabular}{|c|c|c|c|c|c|c|}
\hline Group & $(g)^{3}$ & $(g) \times\left(g^{2}\right)$ & 9 Terms & 12 Terms & Boson & $\mathrm{MSS}+\mathrm{FP}$ \\
\hline 1 & 0 & $\frac{1-D}{2}$ & $\frac{5-2 D}{2}$ & $\frac{2}{3}(3-D)$ & $\frac{30-13 D}{6}$ & $-r$ \\
\hline 2 & $\frac{D-3}{3}$ & $\frac{1}{2}$ & $\frac{D-3}{2}$ & 0 & $\frac{5 D-12}{6}$ & $\frac{r+1}{3}$ \\
\hline 3 & 1 & $\frac{D-3}{2}$ & $\frac{1}{2}$ & $\frac{D-3}{3}$ & $\frac{5 D-6}{6}$ & $\frac{r}{2}$ \\
\hline 4 & $-\frac{1}{3}$ & 0 & 0 & $\frac{3-D}{3}$ & $\frac{2-D}{3}$ & $-\frac{r}{6}$ \\
\hline 5 & 0 & $\frac{1}{2}$ & $\frac{D-3}{2}$ & $\frac{2 D-6}{3}$ & $\frac{7 D-18}{6}$ & $\frac{r}{2}$ \\
\hline
\end{tabular}
for $D=6$.
This completes our proof of (1), (2), and (3). It is curious that we have not had to invoke the gauge condition, which was needed in [14], in this proof.

\section{A POTENTIAL ALGORITHM TO GENERATE THE MAP TO THIRD ORDER AND BEYOND}

In this section we outline an algorithmic approach to determining the map $T_{g}$. This involves perturbatively generating higher order expressions in a manner reminiscent of that in [8]. However, the approach presented here comes with the potential advantage of yielding the map directly as opposed to generating the inverse map $T_{g}^{-1}$.

As mentioned already below Eq. (8), we first note that the structure of the map presented in this paper is simple compared to that in [14]. The entire map in (8), at order $g^{3}$, involves a single structure. We present below an algorithm that generates exactly this structure and suggesting a simple all-order generalization of our results.

We start by noting that the "base" structure - the order $g$ result-has the form: $\partial C A A$. Our claim is that there exists a realization of the map $T_{g}$, to all orders, generated entirely by linking a series of $\partial C A$ structures to this base structure. 
We illustrate this first at order $g^{2}$. The map, at this order, requires one $\partial C A$ in addition to the base structure.

$$
\mathcal{T}\left(g^{2}\right) \sim g^{2} \partial C A \partial C A A .
$$

We now follow the algorithm below.

(i) Sprinkle Lorentz indices on the base $\partial C A A$ block, such that the indices are all distinct. A set of three terms having the same "external" structure but with the three indices on the base-block permuted cyclically constitute a "triplet."

(ii) Choose the two Lorentz indices on the first "block" to be different, for example without loss of generality we can choose them to be $\rho$ and $\lambda$ respectively.

(iii) Discard all terms with $\mu$ on the $\partial$ of the first block (Note that $A_{\mu} \square$ acting on such terms trivially vanish). For

$$
\mathcal{T}\left(g^{2}\right) \sim g^{2} \partial C A \partial C A A,
$$

we distribute Lorentz indices on the $\partial C A A$ block. We have three sets of indices at this order: $\rho, \lambda$ which are summed over and $\mu$ which is the free index. There is only one "triplet" possible at this order, with $\mu, \rho$ and $\lambda$ all sprinkled on the last block. Hence, at this order, the algorithm generates three terms in the map $T_{g}$ :

$$
T_{g}: g^{2} \partial_{\rho} C A_{\lambda} \partial_{[\rho} C A_{\lambda} A_{\mu]} .
$$

Moving to order $g^{3}$, our procedure asks that we add two $\partial C A$ structures to the base structure. So we have

$$
O\left(g^{3}\right)=g^{3} \partial C A \partial C A \partial C A A .
$$

We again distribute Lorentz indices on the $\partial C A A$ block. At this order, we have four sets of indices to work with: $\rho, \sigma, \lambda$ all summed over and $\mu$ which is free. There are 4 ways of selecting 3 different indices (triplets) from the available set. Without loss of generality we choose the Lorentz indices on the first block to be $\rho$ and $\lambda$ respectively. This leaves us with two indices and two slots, which is two arrangements for each triplet, except for one, where we have the same index ( $\sigma$ in this convention), and hence only one arrangement. This gives us seven triplets, or 21 terms at order $g^{3}$, and the map

$$
\begin{aligned}
T_{g}: g^{3} & \partial_{\rho} C A_{\lambda} \partial_{\lambda} C A_{\sigma} \partial_{[\mu} C A_{\rho} A_{\sigma]} \\
& \partial_{\rho} C A_{\lambda} \partial_{\sigma} C A_{\lambda} \partial_{[\mu} C A_{\rho} A_{\sigma]} \\
& \partial_{\rho} C A_{\lambda} \partial_{\mu} C A_{\sigma} \partial_{[\sigma} C A_{\rho} A_{\lambda]} \\
& \partial_{\rho} C A_{\lambda} \partial_{\sigma} C A_{\mu} \partial_{[\sigma} C A_{\rho} A_{\lambda]} \\
& \partial_{\rho} C A_{\lambda} \partial_{\rho} C A_{\sigma} \partial_{[\sigma} C A_{\lambda} A_{\mu]} \\
& \partial_{\rho} C A_{\lambda} \partial_{\sigma} C A_{\rho} \partial_{[\sigma} C A_{\lambda} A_{\mu]} \\
& \partial_{\rho} C A_{\lambda} \partial_{\sigma} C A_{\sigma} \partial_{[\mu} C A_{\lambda} A_{\rho]},
\end{aligned}
$$

exactly matching the structures that appear in (8).
This algorithm generates the terms in sets that conveniently satisfy the gauge condition. The coloured sets above satisfy the gauge constraint when taken together, while the uncolored ones satisfy the gauge constraint by themselves. However, although this algorithm reproduces the map structure correctly, it does not predict the overall coefficients in front of these terms. These need to be determined by the calculations for the three checks. For example, the coefficient of one set of terms is fixed by the requirement that it

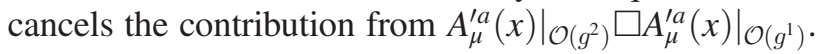

It is fairly straightforward to write down the structures expected at order $g^{4}$, following this algorithm, however performing the relevant checks becomes technically involved.

We conclude that (8) represents an alternate Nicolai map in six dimensions, up to $\mathcal{O}\left(g^{3}\right)$, distinct from the map in [14]. This raises the possibility that there exists a dimension-dependent map that differs for each critical dimension. However, we note that the checks to this order for this particular map do not guarantee that this map will work at next/higher order. ${ }^{2}$ The result in [14] is different because it is derived from the R-prescription and is limited to $\mathcal{O}\left(g^{3}\right)$ only because the procedure becomes technically involved at higher orders.

There is a third and rather unlikely outcome: that six dimensions is special for yet unknown reasons. For another curious result within this formalism that singles out six dimensions, see Eq. (3.10) in [19]. $D=6$ is also home to the mysterious $N=(2,0)$ theory [20-23] which still lacks a complete Lagrangian description [24].

A natural question to ask is how the symmetries in the theory are reflected in the new formalism. How supersymmetry is captured by the resulting bosonic theory was discussed in [19], where explicit calculations were performed for $N=4$ super-Yang-Mills theory. In particular, it was shown that correlators from the standard perturbative computation agree entirely with the corresponding correlators that result from the "mapped" theory. A related issue, that we hope to return to, is the relationship between division algebras and supersymmetry [25] within the framework of the map.

There is an intriguing relationship between supersymmetric theories that admit Nicolai maps and integrable systems. In particular, the existence of integrable structures such as Backlund transformations for supersymmetric Liouville and sine-Gordon theories, and their ties with Nicolai maps [26] is curious and deserving of further study.

\section{ACKNOWLEDGMENTS}

We are grateful to Hermann Nicolai for detailed discussions. We thank Olaf Lechtenfeld for comments and correspondence.

\footnotetext{
${ }^{2}$ If this map survives to higher orders, the gauge condition may become necessary, in keeping with [14].
} 


\section{APPENDIX: JACOBIAN CALCULATION}

We present below a part of the calculation referred to below Eq. (28).

\section{First set of terms at $\mathcal{O}\left(g^{3}\right)$}

These are the details for the first nine terms in (8).

\section{a. Line 3 in $(8)$}

Functional differentiation of the middle block field in each of the first three lines yields

$$
\begin{aligned}
\frac{\delta A_{\mu}^{a \prime}(x)}{\delta A_{\nu}^{p}(v)}= & \frac{1}{2} g^{3} f^{a b c} f^{b d e} f^{d m n} \int d y d z d w \partial_{\rho} C(x-y) A_{\lambda}^{c}(y) \partial_{\lambda} C(y-z) \delta_{\sigma}^{\nu} \delta^{e p} \delta(z-v) \\
& \times\left\{\partial_{\mu} C(z-w) A_{\rho}^{m}(w) A_{\sigma}^{n}(w)+\partial_{\rho} C(z-w) A_{\sigma}^{m}(w) A_{\mu}^{n}(w)+\partial_{\sigma} C(z-w) A_{\mu}^{m}(w) A_{\rho}^{n}(w)\right\},
\end{aligned}
$$

tracing over here involves setting $\mu=\nu, a=p, x=v$ and integrating over $x$. This is then

$$
\begin{aligned}
& \frac{1}{2} g^{3} f^{a b c} f^{b d a} f^{d m n} \int d x d y d w \partial_{\rho} C(x-y) A_{\lambda}^{c}(y) \partial_{\lambda} C(y-x) \\
& \quad \times\left\{\partial_{\mu} C(x-w) A_{\rho}^{m}(w) A_{\mu}^{n}(w)+\partial_{\rho} C(x-w) A_{\mu}^{m}(w) A_{\mu}^{n}(w)+\partial_{\mu} C(x-w) A_{\mu}^{m}(w) A_{\rho}^{n}(w)\right\} .
\end{aligned}
$$

The first and third terms above cancel against each other while the middle terms vanishes (symmetry argument) so these three lines do not contribute to the Jacobian trace.

\section{b. Line 4 in (8)}

After Functional differentiation and tracing over we have

$$
\begin{aligned}
& \frac{1}{2} g^{3} f^{a b c} f^{b d a} f^{d m n} \int d x d y d w \partial_{\rho} C(x-y) A_{\lambda}^{c}(y) \partial_{\sigma} C(y-x) \\
& \quad \times\left\{\partial_{\sigma} C(x-w) A_{\rho}^{m}(w) A_{\lambda}^{n}(w)+\partial_{\rho} C(x-w) A_{\lambda}^{m}(w) A_{\sigma}^{n}(w)+\partial_{\lambda} C(x-w) A_{\sigma}^{m}(w) A_{\rho}^{n}(w)\right\} .
\end{aligned}
$$

Note that $\partial_{\sigma}^{y} C(y-x)=-\partial_{\sigma}^{x} C(x-y)$ meaning that the first line above is symmetric in $\rho, \sigma$ while the bracket is antisymmetric in the same two indices. Hence this contribution vanishes.

\section{c. Line 5 in (8)}

After differentiating and tracing this reads

$$
\begin{aligned}
& \frac{1}{2} g^{3} f^{a b c} f^{b d a} f^{d m n} \int d x d y d w \partial_{\rho} C(x-y) A_{\lambda}^{c}(y) \partial_{\rho} C(y-x) \\
& \quad \times\left\{\partial_{\mu} C(x-w) A_{\lambda}^{m}(w) A_{\mu}^{n}(w)+\partial_{\lambda} C(x-w) A_{\mu}^{m}(w) A_{\mu}^{n}(w)+\partial_{\mu} C(x-w) A_{\mu}^{m}(w) A_{\lambda}^{n}(w)\right\} .
\end{aligned}
$$

These three terms vanish by the same arguments that applied to the terms in line 4 of (8).

\section{Second set of terms at $\mathcal{O}\left(g^{3}\right)$}

We have twelve remaining terms in (8). Functional differentiation and trace in the middle block yields

$$
\begin{aligned}
& \frac{3-D}{3} g^{3} f^{a b c} f^{b d a} f^{d m n} \int d x d y d w \partial_{\rho} C(x-y) A_{\lambda}^{c}(y) \partial_{\sigma} C(y-x) \\
& \quad \times\left\{\partial_{\sigma} C(x-w) A_{\rho}^{m}(w) A_{\lambda}^{n}(w)+\partial_{\rho} C(x-w) A_{\lambda}^{m}(w) A_{\sigma}^{n}(w)+\partial_{\lambda} C(x-w) A_{\sigma}^{m}(w) A_{\rho}^{n}(w)\right\} .
\end{aligned}
$$

These three term vanish by using $\partial_{\sigma}^{y} C(y-x)=-\partial_{\sigma}^{x} C(x-y)$ as the first line above is symmetric in $\rho, \sigma$ while the bracket is antisymmetric in the same two indices. So this contribution vanishes. 
[1] H. Nicolai, On a new characterization of scalar supersymmetric theories, Phys. Lett. 89B, 341 (1980).

[2] H. Nicolai, Supersymmetric functional integration measures, in Supersymmetry, edited by K. Dietz et al. (Plenum Press, New York, 1984).

[3] T. Matthews and A. Salam, The Green's functions of quantized fields, Nuovo Cimento 12, 563 (1954).

[4] E. Seiler, Schwinger functions for the Yukawa model in two-dimensions with space-time cutoff, Commun. Math. Phys. 42, 163 (1975).

[5] L. D. Faddeev and A. Popov, Feynman diagrams for the Yang-Mills field, Phys. Lett. 25B, 29 (1967).

[6] G. 't Hooft, Renormalization of massless Yang-Mills fields, Nucl. Phys. B33, 173 (1971).

[7] H. Nicolai, Supersymmetry and functional integration measures, Nucl. Phys. B176, 419 (1980).

[8] K. Dietz and O. Lechtenfeld, Nicolai maps and stochastic observables from a coupling constant flow, Nucl. Phys. B255, 149 (1985).

[9] K. Dietz and O. Lechtenfeld, Ghost-free quantisation of non-Abelian gauge theories via the Nicolai transformation of their supersymmetric extensions, Nucl. Phys. B259, 397 (1985).

[10] O. Lechtenfeld, Construction of the Nicolai mapping in supersymmetric field theories, Doctoral thesis, Bonn University, 1984.

[11] R. Flume and O. Lechtenfeld, On the stochastic structure of globally supersymmetric field theories, Phys. Lett. 135B, 91 (1984).

[12] S. Ananth, H. Nicolai, C. Pandey, and S. Pant, Supersymmetric Yang-Mills theories: Not quite the usual perspective, J. Phys. A 53, 174001 (2020).
[13] L. Brink, J. H. Schwarz, and J. Scherk, Supersymmetric Yang-Mills theories, Nucl. Phys. B121, 77 (1977).

[14] S. Ananth, O. Lechtenfeld, H. Malcha, H. Nicolai, C. Pandey, and S. Pant, Perturbative linearization of supersymmetric Yang-Mills theory, J. High Energy Phys. 10 (2020) 199.

[15] V. de Alfaro, S. Fubini, and G. Furlan, Stochastic identities in the light cone gauge, Phys. Lett. 163B, 176 (1985).

[16] V. de Alfaro, S. Fubini, G. Furlan, and G. Veneziano, Stochastic identities in quantum theory, Nucl. Phys. B255, 1 (1985).

[17] S. Ananth, L. Brink, and M. Mali, Yang-Mills theories and quadratic forms, J. High Energy Phys. 08 (2015) 153.

[18] S. Ananth, C. Pandey, and S. Pant, Higher spins, quadratic forms and amplitudes, J. High Energy Phys. 07 (2020) 100.

[19] H. Nicolai and J. Plefka, $N=4$ super-Yang-Mills correlators without anticommuting variables, Phys. Rev. D 101, 125013 (2020).

[20] W. Nahm, Supersymmetries and their representations, Nucl. Phys. B135, 149 (1978).

[21] E. Witten, Some comments on string dynamics, arXiv:hepth/9507121.

[22] A. Strominger, Open p-branes, Phys. Lett. B 383, 44 (1996).

[23] G. Moore, http://www.physics.rutgers.edu/ gmoore/ FelixKleinLectureNotes.pdf.

[24] S. Ananth, S. Kovacs, Y. Sato, and H. Shimada, Towards a tensionless string field theory for the $\mathcal{N}=(2,0)$ CFT in $d=6$, J. High Energy Phys. 07 (2018) 135.

[25] A. Anastasiou, L. Borsten, M. J. Duff, L. J. Hughes, and S. Nagy, Super Yang-Mills, division algebras and triality, J. High Energy Phys. 08 (2014) 080.

[26] D. Birmingham, M. Blau, M. Rakowski, and G. Thompson, Topological field theory, Phys. Rep. 209, 129 (1991). 